\title{
NÍVEL DE CONHECIMENTO DOS ACADÊMICOS DE FISIOTERAPIA SOBRE A ATENÇÃO BÁSICA VOLTADA À INFÂNCIA E À ADOLESCÊNCIA
}

\author{
Simone de Paula ${ }^{1}$, Caren Lara Martins Picasso ${ }^{2}$
}

\begin{abstract}
Resumo: A formação generalista do fisioterapeuta baseada em preceitos humanistas e inovadores constitui-se uma prioridade nos tempos atuais. As ações pedagógicas durante a formação superior devem privilegiar a relação da teoria com a prática em todos os espaços de atuação em saúde. No âmbito da infância e da adolescência, as políticas públicas nacionais destacam que este ciclo de vida é um campo prioritário dentro dos cuidados à saúde da população, especialmente através de estratégias de promoção à saúde e prevenção de agravos. O objetivo deste estudo foi avaliar o nível de conhecimento e a percepção dos acadêmicos sobre o papel da fisioterapia na atenção básica voltada para a infância e a adolescência. $\mathrm{O}$ estudo caracterizou-se por uma pesquisa descritiva, do tipo exploratória e com abordagem quantiqualitativa. A amostra da pesquisa foi composta por acadêmicos regularmente matriculados no curso de fisioterapia de uma universidade, de ambos os sexos e com idade igual ou superior a 18 anos. A coleta de dados foi realizada através de um questionário misto contendo informações sobre idade, semestralidade e perguntas abertas (duas) e fechadas (seis) que contemplavam itens, como a formação, o interesse e o conhecimento sobre a fisioterapia na atenção básica voltada para a infância e adolescência. O questionário online foi elaborado através da ferramenta do Google Forms ${ }^{\circledR}$, disponível aos acadêmicos no período de julho a setembro de 2016. A amostra do estudo constituiu-se de 19 acadêmicos, com idade média de 26,85 anos $( \pm 5,9)$, em sua maioria, do gênero feminino entre o $7^{\circ}$ e $10^{\circ}$ semestre $(68,4 \%)$. A opção pelo futuro setor de trabalho (público, privado ou ambos), 11 alunos $(57,9 \%)$ responderam que desejavam trabalhar em ambos os serviços, 4 (21,1\%) no setor público e $2(10,5 \%)$ no setor privado. A maioria dos estudantes mostrou um bom nível de conhecimento sobre os conceitos básicos de promoção em saúde. $\mathrm{O}$ acadêmico $\mathrm{A}$, refere que "a atenção básica voltada para a criança e adolescente tem como intuito a prevenção e a promoção da saúde por meio de ações educativas". É importante ressaltar que, apesar dos significativos depoimentos, dos 19 acadêmicos entrevistados somente 3 apontam uma visão diferenciada sobre a atuação fisioterapêutica na atenção primária. O acadêmico A diz que "a fisioterapia pode atuar na área preventiva e educativa, não devendo ficar limitada apenas a área de reabilitação". Alguns relatos complementam a carência de especificidade destas ações e ilustram apenas a temática a ser trabalhada, tais como, a importância dos exercícios físicos e da boa postura. Poucos alunos destacaram a importância da ludicidade nas ações em saúde na infância e adolescência. Ainda, $73,7 \%$ dos acadêmicos entrevistados nesta pesquisa consideram que a fisioterapia é muito importante para a atenção básica voltada à criança e ao adolescente. Ressalta-se que a inserção do fisioterapeuta em serviços públicos de saúde ainda é um processo de construção, o que pode justificar a dificuldade dos acadêmicos em detalhar as ações e funções práticas do fisioterapeuta no contexto da atenção primária.
\end{abstract}

Palavras-chave: Fisioterapia. Educação em Saúde. Promoção da Saúde.

1 Fisioterapeuta, Doutora em Saúde da Criança, Docente do Curso de Fisioterapia da Universidade Feevale.

2 Fisioterapeuta, Mestre em Gerontologia Biomédica, Docente do Curso de Fisioterapia da Universidade Feevale. 


\title{
LEVEL OF PHYSIOTHERAPY KNOWLEDGE IN PRIMARY CARE DIRECTED TO CHILDHOOD AND ADOLESCENCE
}

\begin{abstract}
General physiotherapist education based on humanistic and innovative principles is a priority these days. The educational measures during higher education should focus on the relationship between theory and practice in all health-related areas. Within the context of childhood and adolescence, national public policies emphasize that this life cycle is a major field within the population's health care, in particular, through strategies that promote health and prevent disease. The goal was to assess the student's level of knowledge and perception regarding the role of physiotherapy in primary care intended for children and adolescents. The study featured a descriptive and exploratory research with both a quantitative and qualitative approach. The research sample consisted of a group of students enrolled in the physiotherapy course of a given University, of both genders, aged 18 or over. Data was gathered through a mixed survey containing information on age, number of completed semesters and (two) open and (six) closed questions that contemplated items such as: physiotherapy training, interest and knowledge drawn toward primary care intended for childhood and adolescence. The online questionnaire was prepared using the Google Forms ${ }^{\circledR}$ tool, made available to students during the months of July to September 2016. The study sample consisted of 19 students, with an average age of 26.85 years ( \pm 5.9 ), mostly female and having completed around 7 to 10 semesters (68.4\%). In respect of the future work sector (public, private or both) option, 11 students $(57.9 \%)$ answered that they wanted to work in both services, $4(21.1 \%)$ said they wanted to work in the public sector and $2(10.5 \%)$ said they wanted to work in the private sector. Most students revealed having a good level of knowledge about the basic concepts of promoting health. Student A, states that "primary care targeted towards children and adolescents is intended as a preventive measure, as well as promoting health through educational measures". It should be emphasized that, despite the significant testimonies, of the 19 students interviewed, only 3 seemed to have a diferente opinion regarding physiotherapy performance in primary care. Student A says that "physiotherapy can act as a measure of prevention as well as educational, and it should not be limited to the field of rehabilitation." Some reports complement the lack of specificity in these measures and only show the subject to be worked upon, such as the importance of exercising and having a good posture. Only a few students highlighted the importance of playfulness in the measures intended for childhood and adolescence. Nevertheless, $73.7 \%$ of all students interviewed during this study consider that physiotherapy is very important for primary care focused on children and adolescents. It is emphasized that the integration of a physiotherapist in public health services is still in its early stages, which may explain the difficulties that students have in detailing the measures and practical roles of a physiotherapist within the context of primary care.
\end{abstract}

Keywords: Physical Therapy Specialty. Health Education. Health Promotion.

\section{INTRODUÇÃO}

O Conselho Federal de Fisioterapia define que a atuação do fisioterapeuta deve abranger o desenvolvimento de ações em todos os níveis de atenção à saúde, em consonância com as diretrizes do Sistema Único de Saúde (SUS) e fundamentado nos princípios da universalidade, integralidade e equidade. Neste contexto, a formação generalista do fisioterapeuta baseada em preceitos humanistas e inovadores constituise de uma prioridade nos tempos atuais. As ações pedagógicas durante a formação superior devem privilegiar a relação da teórica com a prática em todos os espaços de atuação em saúde. A fisioterapia, ao longo de sua história, atuou de modo prioritário no nível terciário, destinando-se à reabilitação de sequelas e complicações. No 
entanto, de acordo com Bispo Júnior (2009) a atual formação acadêmica visando a aproximação da área da fisioterapia com as demandas da população não pode ocorrer fundamentada nas práticas de atuação profissional exclusivamente reabilitadoras. Em virtude destas perspectivas, as Diretrizes Curriculares Nacionais para o ensino de graduação em fisioterapia destacam que a formação do fisioterapeuta deve contemplar as necessidades sociais da saúde, com ênfase nos pilares do SUS (BISPO JUNIOR, 2009; CONSELHO NACIONAL DE EDUCAÇÃO, 2001). No âmbito da infância e da adolescência, as políticas públicas nacionais destacam que este ciclo de vida é um campo prioritário dentro dos cuidados à saúde da população, especialmente através de estratégias de promoção à saúde e prevenção de agravos (BRASIL, 2009). Além disso, a Constituição Federal de 1988 e a Lei n. 8.069 de 13 de julho de 1990, denominada Estatuto da Criança e do Adolescente (ECA) considerou que a infância e a juventude são uma prioridade absoluta, merecedoras de proteção especial e integral por parte da família, da sociedade e do Estado (OLIVA; KAUCHAKIE, 2009). Apesar da evidente necessidade de mudanças na formação acadêmica dos profissionais da fisioterapia a fim de contemplar as demandas populacionais nesta faixa etária, poucos estudos discutem o conhecimento, as percepções e as perspectivas dos acadêmicos para a atuação em programas e estratégias de atenção básica. Dessa forma, esta pesquisa teve por objetivo avaliar o nível de conhecimento e a percepção dos acadêmicos sobre o papel da fisioterapia na atenção básica voltada para a infância e a adolescência.

\section{METODOLOGIA}

O estudo caracterizou-se por uma pesquisa descritiva, do tipo exploratória e com abordagem quanti-qualitativa. A amostra da pesquisa foi composta por 19 acadêmicos regularmente matriculados no curso de fisioterapia de uma universidade, de ambos os sexos e com idade igual ou superior a 18 anos. Para responder aos objetivos propostos neste estudo, a coleta de dados foi realizada através de um questionário misto contendo informações sobre idade, semestralidade e perguntas abertas (duas) e fechadas (seis) que contemplavam itens, como: a formação, o interesse e o conhecimento sobre a fisioterapia na atenção básica voltada para a infância e adolescência. Os acadêmicos foram contatados pessoalmente ou através de e-mail e redes sociais e convidados a participar voluntariamente da pesquisa. O questionário online foi elaborado através do formulário do Google Forms ${ }^{\circledR}$, disponível aos acadêmicos no período de julho a setembro de 2016. Posteriormente, as informações quantitativas obtidas foram organizadas e os dados tabulados em uma planilha do software Microsoft Office Excel ${ }^{\circledR}$. As variáveis qualitativas foram analisadas através da análise de conteúdo (BARDIN, 2009), resultando na definição dos principais conteúdos e temas das respostas dadas pelos participantes. 


\section{RESULTADOS E DISCUSSÃO}

$\underline{\text { Perfil dos acadêmicos participantes }}$

As principais características dos acadêmicos participantes do estudo são resumidas na Tabela 1. É possível observar que a amostra constituiu-se, em sua maioria, de estudantes do gênero feminino, com idade média de 26,85 anos $( \pm 5,9)$, entre o $7^{\circ}$ e $10^{\circ}$ semestre $(68,4 \%)$. Quanto à opção pelo futuro setor de trabalho (público, privado ou ambos), 11 alunos (57,9\%) responderam que desejavam trabalhar em ambos os serviços, $4(21,1 \%)$ somente no setor público e $2(10,5 \%)$ no setor privado.

Tabela 1. Perfil dos acadêmicos participantes do estudo

\begin{tabular}{l|c|c}
\hline & Frequência & Porcentagem \\
\hline Gênero & & \\
\hline Feminino & 13 & $68,4 \%$ \\
\hline Masculino & 6 & $31,6 \%$ \\
\hline Semestralidade & 0 & \\
\hline Entre $1^{\circ}$ e $3^{\circ}$ & 6 & $0 \%$ \\
\hline Entre $4^{\circ}$ e $6^{\circ}$ & 13 & $68,4 \%$ \\
\hline Entre $7^{\circ}$ e $10^{\circ}$ & & \\
\hline Preferência de áreas de atuação & 4 & $21,1 \%$ \\
\hline Setor público & 2 & $10,5 \%$ \\
\hline Setor privado & 11 & $57,9 \%$ \\
\hline Ambos & 2 & $10,5 \%$ \\
\hline Não sabe informar & &
\end{tabular}

Os resultados do perfil dos acadêmicos são similares a outros estudos (GUEDES, 2008; WERMELINGER et al., 2010; BARRETO, 2014) realizados com diferentes profissionais, demonstrando o interesse de mulheres jovens na formação superior em saúde. O contingente feminino tem-se tornado francamente majoritário na área da saúde, especificamente no período após os anos 70, quando essa participação passou a ser mais expressiva e progressivamente maior.

Em relação às perspectivas de atuação no mercado de trabalho, os universitários participantes desta pesquisa mostraram o crescente interesse pelo setor público, fato também observado no estudo de Pinheiro et al. (2009) que apresentou um percentual de $87,5 \%$ de acadêmicos interessados em ambos os setores. O SUS institucionaliza os serviços de saúde no Brasil, sendo considerado o maior mercado de trabalho em saúde do país. Em virtude disso, a formação do fisioterapeuta integrada aos preceitos das políticas em saúde favorece o comprometimento com o sistema e o engajamento das práticas profissionais (PINHEIRO et al., 2009; ARAÚJO, 2007). Estimativas 
mostram que o setor público gera, aproximadamente, 2 milhões de empregos diretos em saúde (BRASIL, 2003; OLIVEIRA; MACHADO, 2010). Em consonância com as atuais concepções de ensino em saúde e alinhado com as Diretrizes Curriculares Nacionais dos Cursos de Fisioterapia (DCNs), um dos objetivos do curso de fisioterapia da universidade de realização desta pesquisa é formar profissionais capacitados a compreender e analisar as necessidades sociais em saúde, contribuindo para o fortalecimento do SUS (BRASIL, 2002). Neste contexto, o alinhamento entre as diretrizes e princípios do setor público de saúde e o projeto pedagógico do curso pode explicar os resultados encontrados nesta questão.

Conhecimento sobre a atuação fisioterapêutica voltada à saúde da criança e do adolescente

Os acadêmicos selecionados para a pesquisa foram questionados sobre o que sabem ou conhecem em relação à atenção básica voltada para a criança e ao adolescente. Através da análise das respostas no questionário, observou-se que a maioria dos estudantes mostrou um bom nível de conhecimento teórico sobre os conceitos básicos de promoção em saúde. $\mathrm{O}$ acadêmico $\mathrm{A}$, por exemplo, referiu que "a atenção básica voltada para a criança e adolescente tem como intuito a prevenção e a promoção da saúde por meio de açôes educativas (...)". Já o acadêmico B acrescentou que "a criança e o adolescente são prioridade no Estado brasileiro e que devem receber todos os cuidados referentes à sua proteção e desenvolvimento." É importante ressaltar que, apesar dos significativos depoimentos, dos 19 acadêmicos entrevistados somente 3 (três) apontaram uma conceituação diferenciada sobre a atuação fisioterapêutica na atenção primária, englobando uma visão holística e integral sobre as atribuições deste profissional no âmbito preventivo. $\mathrm{O}$ acadêmico A ilustrou o papel do fisioterapeuta em ações de saúde dissertando que "a fisioterapia pode atuar na área preventiva e educativa, não devendo ficar limitada apenas à área de reabilitação".

Grande parte das doenças na infância e na adolescência requer a atuação da fisioterapia. Além da redução na demanda de consultas nas Unidades de Saúde da Família, este profissional atua diretamente em ações preventivas para a diminuição das taxas de morbidade e mortalidade destes grupos etários (DAVID, 2013). Neste contexto, o conhecimento dos profissionais sobre os determinantes sociais da saúde permite compreender melhor as condições que possam levar ao adoecimento, assim como, a elaboração de estratégias preventivas (SÁ; GOMES, 2013). No caso de crianças e adolescentes, também torna-se vital o trabalho de educação para a saúde com os pais e cuidadores, uma vez que esta população ainda é dependente de cuidados externos. Estes aspectos, juntamente com relatos de intervenções específicas voltadas para as condições de saúde sensíveis nesta população, não foram destacadas nas falas dos participantes. Além disso, os resultados encontrados neste estudo expressam o conhecimento dos acadêmicos ainda restrito ao nível secundário e terciário de atenção à saúde. Esse conhecimento limitado restringe a atuação do profissional e destaca o pensamento popular, no qual o fisioterapeuta somente está inserido nos espaços tradicionalmente conhecidos (hospitais e clínicas de reabilitação). 
Sá e Gomes (2013) destacam a crise do modelo terapêutico-curativo frente à mudança epidemiológica nos perfis de morbimortalidade infantil. Apoiada em estratégias baseadas em evidências, esta mudança fala a favor de uma intervenção não invasiva, educadora, o que caracteriza a prática fisioterapêutica. Por ser um cenário atual, é importante considerar que a transformação e a construção da Fisioterapia é complexa e deve começar na graduação e manter-se como um processo de educação continuada (OLIVEIRA et al., 2011). Com base nisso, tem-se enfatizado que a formação do fisioterapeuta seja compatível com as demandas da população e direcionada a desenvolver competências e habilidades gerais para atenção à saúde, como ações de prevenção, promoção e proteção da saúde, além da reabilitação individual e coletiva (BRASIL, 2002).

Em virtude da carência de pesquisas sobre a formação do fisioterapeuta em consonância com os preceitos do SUS, os resultados da literatura ainda são superficiais e controversos. Pinheiro et al. (2009) observaram em seu estudo que os graduandos de fisioterapia demonstraram um conhecimento satisfatório sobre princípios doutrinários e organizacionais do SUS. No entanto, Naves e Brick (2011) mostraram que a maioria dos alunos do curso de fisioterapia de uma universidade paulista tem pouco conhecimento sobre a atuação do fisioterapeuta em saúde pública. Os autores explicam que o escasso número de profissionais nesta área pode justificar o desconhecimento e a falta de interesse dos acadêmicos em relação às necessidades de formação e atuação profissional. Sabe-se da importância da atuação do fisioterapeuta no âmbito da atenção básica, no entanto, essa inserção acontece em pequenos passos (MAIA et al., 2015). Franco (2006) acrescenta que, à medida que os processos de aprendizagem promoverem a identificação dos acadêmicos como sujeitos pertencentes a uma rede com outros sujeitos, provocar-se-á uma cultura diferenciada na produção do cuidado integral.

Em relação às ações que competem ao fisioterapeuta que atua na atenção básica voltada à criança e ao adolescente, algumas afirmações mostraram pouco detalhamento sobre as atividades que podem ser desenvolvidas por este profissional. De forma distante às demandas desta população, o acadêmico $\mathrm{G}$ considerou "importante a parte de ergonomia escolar para estudante e professor". Além disso, alguns relatos complementaram a carência de especificidade destas ações e ilustraram apenas a temática a ser trabalhada, tais como, a importância dos exercícios físicos e da boa postura.

Poucos alunos destacaram a importância da ludicidade nas ações em saúde na infância e adolescência: "As ações para chamar a atenção dos adolescentes devem ser por meio de brincadeiras lúdicas e jogos", escreveu o acadêmico B. De forma similar, outro acadêmico colocou que "podem ser desenvolvidas palestras, jogos, teatros e atividades lúdicas".

As atividades lúdicas fazem parte do cotidiano das pessoas e é uma das estratégias mais eficazes para promover a ação e a aproximação em saúde (GOULART, LUCCHESI, CHIARI; 2010). Coscrato, Pina e Mello (2010) acrescentam ainda que o conceito de educação em saúde vai além da transmissão de informações, configurando 
combinações de experiências de aprendizagem delineadas com vistas a facilitar ações voluntárias conducentes à saúde. Em uma revisão integrativa, os autores mostraram que existem evidências científicas consistentes sobre a eficácia de atividades lúdicas para a promoção de saúde na infância e adolescência.

Além disso, cabe ressaltar que a inserção do fisioterapeuta em serviços públicos de saúde ainda é um processo de construção, o que pode justificar a dificuldade dos acadêmicos do presente estudo em detalhar as ações e atribuições práticas no fisioterapeuta no contexto da atenção primária voltada à saúde da criança e do adolescente.

\section{Importância da fisioterapia na atenção primária}

Apesar de retratar de forma superficial a abordagem prática das ações educativas em saúde, $73,7 \%$ dos acadêmicos entrevistados nesta pesquisa consideraram que a fisioterapia é muito importante para a atenção básica voltada à criança e ao adolescente. De forma similar aos nossos achados, Naves e Brick (2011), que mostraram que os alunos consideram importante a atuação do fisioterapeuta no SUS, assim como a preparação e a qualificação do atendimento em saúde coletiva. São competências do fisioterapeuta o planejamento, a programação, a coordenação, a execução e a supervisão da aplicação de métodos e técnicas que visem à promoção da saúde, seja no nível de atenção básica, secundária ou terciária, englobando o paciente em todos os seus aspectos: físicos, emocionais e culturais (DAVID et al., 2013). Portanto, sua formação universitária não deve se limitar somente a recuperar, reabilitar ou atenuar os comprometimentos provocados pelas patologias de base, mas o capacita também a avaliar, prevenir e trabalhar em promoção a saúde.

A atuação do fisioterapeuta na rede básica de saúde por meio das três estratégias de intervenção - orientação, assistência e acompanhamento - fornece uma perspectiva ampla e integral do paciente pediátrico, viabilizando uma terapêutica multiprofissional mais efetiva (SÁ; GOMES, 2013). Schlessman et al. (2011) acrescenta que, além da competência clínica, o fisioterapeuta deve possuir expertise em cuidados primários a fim reconhecer e atender as necessidades no espaço de cuidado, buscando uma ação que permita desenvolver as potencialidades dos indivíduos.

A população infantil é um dos grupos etários mais vulneráveis e é assistido por meio de políticas através do programa de saúde da criança e do adolescente. Este programa tem como responsabilidades a garantia dos aspectos nutricionais, reforço constante quanto ao aleitamento materno, acompanhamento regular do crescimento e do desenvolvimento da criança, imunização por meio de campanhas de vacinação, e ainda assistência às doenças prevalentes, como diarreia e infecções respiratórias agudas (DAVID et al., 2013).

$\mathrm{Na}$ área da saúde do adolescente, o Ministério da Saúde desenvolveu o Programa de Saúde do Adolescente. O Programa elegeu como áreas prioritárias de atuação o crescimento e o desenvolvimento, a sexualidade, a saúde bucal, a saúde mental, a saúde 
reprodutiva, a saúde do escolar adolescente e a prevenção de acidentes, fundamentadas nos princípios da integralidade, multidisciplinaridade e intersetorialidade, associada a uma política de promoção da saúde, desenvolvimento de práticas educativas, identificação de grupos de riscos, detecção precoce dos agravos, tratamento e reabilitação (BRASIL, 2005).

David et al. (2013) acrescenta que, quando direta, a atuação do fisioterapeuta programa retornos periódicos nos quais ele realiza a avaliação do desenvolvimento e do crescimento da criança, analise aspectos sensoriais e motores, reforça orientações e cuidados quanto à doenças respiratórias e o manejo de pacientes crônicos. Através desta atuação, este profissional avalia a evolução da criança e do adolescente, identifica precocemente alterações motoras e posturais, agudizações do quadro respiratório e, ainda, monitora as condições domiciliares.

Neste contexto, a inclusão deste profissional na atenção primária é de significativa importância uma vez que a intervenção deste profissional pode beneficiar diretamente vários setores da sociedade, incluindo a atuação na infância e adolescência. Já existem relatos significativos na literatura científica que demonstram as diversas possibilidades de ações em saúde coletiva promovidas por este profissional. O fisioterapeuta pode realizar o acompanhamento regular na primeira infância e as avaliações fisioterapêuticas programadas que permitem detectar precocemente atrasos ou desvios. Ele também pode orientar os pais sobre as características da criança, ensinar princípios básicos de estimulação sensório-motora e anotar dados sobre o crescimento e desenvolvimento das crianças acompanhadas nas Unidades de Saúde da Família através da caderneta infantil, promovendo a vigilância à saúde infantil. Além disso, o fisioterapeuta pode estabelecer formas de monitorar a criança, seja através da programação de retornos semanais às unidades de saúde ou por meio de visitas domiciliares (DAVID et al., 2013; BISPO JUNIOR, 2014; MAIA et al., 2015).

\section{CONSIDERAÇÕES FINAIS}

Os resultados do presente estudo mostraram que os acadêmicos de fisioterapia apresentam um bom nível de conhecimento sobre a atuação do fisioterapeuta na atenção primária voltada à infância e à adolescência, apesar do limitado domínio sobre as práticas a serem desenvolvidas. O estudo também demonstrou elevado percentual de universitários com interesse em atuar em ambos os setores da saúde (público e privado), reforçando as necessidades de avanço e desenvolvimento de estratégias de ensino-aprendizagem que estejam em consonância com os princípios do Sistema Único de Saúde para uma atuação integral e plena na infância e adolescência. Apesar das potencialidades apresentadas neste estudo pelas narrativas dos acadêmicos participantes, algumas lacunas de aprendizagem ainda podem ser observadas no processo de formação do fisioterapeuta. Uma delas diz respeito a necessidade de ampliação da abordagem fisioterapêutica para além do modelo biomédico, possibilitando a inserção de intervenções educativas e preventivas na prática fisioterapêutica, atendendo às demandas 
individuais e coletivas deste grupo etário. $\mathrm{O}$ segundo aspecto a ser desenvolvido é o estabelecimento de abordagens específicas voltadas para a criança e o adolescente, que ainda carecem de estudos aprofundados e adequados às peculiaridades desta população. Por fim, o processo de formação e identificação do fisioterapeuta com a promoção da saúde voltada para a infância e adolescência devem ser enfatizadas nas propostas curriculares vigentes. É importante considerar que o estudo apresenta limitações. $\mathrm{O}$ pequeno número amostral e o instrumento utilizado justificam a elaboração e o desenvolvimento de mais pesquisas na área a fim de aprofundar as percepções e os conhecimentos destes profissionais em formação para sua a participação na equipe multiprofissional, de forma transdisciplinar e horizontal.

\section{REFERÊNCIAS}

ARAÚJO, Dolores; MIRANDA, Maria Cláudia Gomes de; BRASIL, Sandra. Formação de profissionais da saúde na perspectiva da integralidade. Rev. Baiana de Saúde Pública, v. 31, p. 20-31, 2007.

BARDIN, L. Análise de conteúdo. Lisboa: Edições 70, 2009.

BARRETO, Andreia. A mulher no Ensino Superior: distribuição e representatividade. Cadernos do GEA, n. 6, jul.-dez., 2014.

BISPO JÚNIOR, José Patrício. Formação em fisioterapia no Brasil: reflexões sobre a expansão do ensino e os modelos de formação. História, Ciências, Saúde, v.16, n.3, p.655-668, jul.-set. 2009.

. Fisioterapia e Saúde Coletiva. São Paulo: Hucitec, 2014.

BRASIL. Ministério da Educação. Conselho Nacional de Educação Câmara de Educação Superior. Resolução CNE/CES 4, de 19 de fevereiro de 2002. Institui Diretrizes Curriculares Nacionais do Curso de Graduação em Fisioterapia. Diário Oficial União. Disponível em: <http://portal.mec.gov.br/cne/arquivos/pdf/CES042002.pdf > Acesso em: abr. 2014.

Ministério da Saúde. Observatório de recursos humanos em saúde no Brasil: estudos e análises. Rio de Janeiro: Editora Fiocruz, 2003, 460p.

. Ministério da Saúde. Saúde integral de adolescentes e jovens: orientações para organização de serviços de saúde. Brasília: Ministério da Saúde; 2005.

. Ministério da Saúde. Secretaria de Políticas de Saúde. Área de Saúde da Criança: Crescimento e Desenvolvimento. Brasília, 2009.

CONSELHO NACIONAL DE EDUCAÇÃO. Diretrizes Curriculares Nacionais dos cursos de graduação em Fisioterapia, Fonoaudiologia e Terapia Ocupacional. Brasília, 2001.

COSCRATO, Gisele; PINA, Juliana Coelho; MELLO, Débora Falleiros. Utilização de atividades lúdicas na educação em saúde: uma revisão integrativa da literatura. Acta Paul Enferm, v.23, n.2, p.257-63, 2010. 
DAVID, Maria Laura Oliveira et al. Proposta de atuação da fisioterapia na saúde da criança e do adolescente: uma necessidade na atenção básica. Saúde debate [online], vol.37, n.96, p.120$129,2013$.

FRANCO, Túlio Batista. As Redes na Micropolítica do Processo de Trabalho em Saúde. Rio de Janeiro: CEPESC-IMS/UERJ-ABRASCO, 2006

GUEDES, Moema de Castro. A presença feminina nos cursos universitários e nas pósgraduações: desconstruindo a ideia da universidade como espaço masculino. Hist. cienc. Saude-Manguinhos, Rio de Janeiro, v. 15, p. 117-132, 2008.

GOULART, Bárbara NG.; LUCCHESI, Millena Citon; CHIARI, Brasilia Maria. A unidade básica de saúde como espaço lúdico para educação e promoção da saúde infantil. Rev Bras Crescimento Desenvolvimento Hum., v. 20, n. 3, p. 757-761, 2010.

MAIA, Francisco Eudison da Silva et al. A importância da inclusão do profissional fisioterapeuta na atenção básica de Saúde. Revista da Faculdade de Ciências Médicas de Sorocaba. ISSN eletrônico 1984-4840, [S.1.], v. 17, n. 3, p. 110-115, set. 2015.

NAVES, Cristiane Roberta; BRICK, Vanessa de Souza. Análise quantitativa e qualitativa do nível de conhecimento dos alunos do curso de fisioterapia sobre a atuação do fisioterapeuta em saúde pública. Ciência \& Saúde Coletiva, v.16, suplemento 1, p. 1525-1534. 2011.

OLIVA, Jimena Cristina Gomes Aranda; KAUCHAKJE, Samira. As políticas sócias públicas e os novos sujeitos de direitos: crianças e adolescentes. Rev. Katál Florianópolis, v. 12, n.1, p.22-31, jan./jun. 2009.

OLIVEIRA, Eliane dos Santos de; MACHADO, Maria Helena. Para Medir o Trabalho em Saúde no Brasil: Principais Fontes de Informações. Divulgação em Saúde para Debate, Rio de Janeiro, n. 45, p. 105-125, 2010.

OLIVEIRA, Greicimar de, et al. Conhecimento da equipe de saúde da família acerca da atuação do fisioterapeuta na atenção básica. Rev Bras Promoç Saúde, n. 24, v.4, out./dez., 2011.

PINHEIRO, Liane Barreto Diógenes; DIÓGENES, Paula Nogueira; FILGUEIRAS, Marcelo de Carvalho et al. Conhecimento de graduandos em fisioterapia na Universidade de Fortaleza sobre o Sistema Único de Saúde. Fisioter Pesq., v.16, n.3, p.211-6, 2009.

SÁ, Miriam Ribeiro Calheiros de; GOMES, Romeu. A promoção da saúde da criança: a participação da Fisioterapia. Ciênc. saúde coletiva, Rio de Janeiro , v. 18, n. 4, p. 1079-1088, 2013.

SCHLESSMAN Amy et al. The role of Physical Therapists in pediatric health promotion and obesity prevention: comparison of attitudes. Pediatr Phys Ther, n. 23, v. 1, p. 79-86, 2011.

WERMELINGER, Monica et al. Análise da força de trabalho do setor saúde no Brasil: focalizando a feminização. Revista Divulgação em Saúde para Debate, n.45, p. 54-70, 2010. 